\title{
Sympathetic Axons Pathfind Successfully in the Absence of Target
}

\author{
G. Guidry and S. C. Landis \\ Department of Neuroscience, Case Western Reserve University School of Medicine, Cleveland, Ohio 44106-4975
}

To determine whether sympathetic axons require the presence of a peripheral target to grow to the correct destination, we examined the developing footpad innervation in tabby mutant mice which lack sweat glands. Despite the absence of sweat glands, noradrenergic sympathetic axons are transiently present in the presumptive target area and avoid the more distal epidermal/dermal domain occupled by sensory axons. Since sympathetic axon pathfinding was not dependent upon the target tissue, we compared the subsequent development of sweat gland axons in tabby footpads with that in control footpads. In wild-type mice, the gland-associated axonal plexus expands considerably as the secretory tubule enlarges and coils. This expansion, however, does not occur in tabby mice. The sweat gland innervation of wild-type mice loses catecholamines and acquires AChE activity and vasoactive intestinal peptide immunoreactivity. In tabby mutant mice, catecholaminergic fibers remain in the glandless footpads for 2 weeks and fail to acquire AChE or vasoactive intestinal peptide. In contrast to the altered development of gland innervation in tabby, the development of the innervation of footpad blood vessels was unaffected. Our observations indicate that the target is not required to direct sympathetic axons to the presumptive gland region of the footpad. In the absence of the target tissue, however, gland-targeted sympathetic axons retain an immature morphology and transmitter phenotype and then disappear.

[Key words: sympathetic neuron development, axon guidance, neurotransmitter phenotype, synapse formation, neuron survival, NGF]

During development, growing axons accurately pathfind long distances to appropriate target tissues (Landmesser, 1984; Goodman and Shatz, 1993; O'Leary and Koester, 1993). While it seems unlikely that targets provide guidance cues when growth cones are distant, they can provide local cues. In the CNS, culture and in vivo studies revealed the importance of tropic influences in guiding commissural axons in spinal cord (Tessier-Lavigne et al., 1988; Bovolenta and Dodd, 1991; Yaginuma and Oppenheim, 1991) and in eliciting collateral extension by cortical axons in brainstem (Heffner et al., 1990; O'Leary et al., 1990). In culture, target tissues attract axons from spinal cord

Received May 11, 1995; revised July 17, 1995; accepted July 20, 1995.

We thank Dr. Mahendra Rao, who participated in initial stages of this project, and Carolyn Marks, who helped with the morphological analysis. This work was supported by Javits Neuroscience Investigator Award NS 023678. S.C.L. is a MacKnight Neuroscience Senior Investigator.

Correspondence should be addressed to Dr. Story Landis at the above address.

Copyright (C) 1995 Society for Neuroscience $0270-6474 / 95 / 157565-10 \$ 05.00 / 0$ or appropriate sensory ganglia (Lumsden and Davies, 1986; McÇaig, 1986). Consistent with a role for target-derived cues in the peripheral nervous system, muscle nerve branches fail to form when muscle is ablated by somite irradiation, although not following somite extirpation (Lewis et al., 1981; Phelan and Hollyday, 1990). Removal of axial muscle precursors results in failed myotomal nerve formation (Tosney, 1987; Phelan and Hollyday, 1990). Ablation, transplantation and genetic studies indicate that motor axons are attracted to the base of pectoral fins in medaka (Okamoto and Kuwada, 1991a,b). In sum, these studies provide evidence that target tissues play a role in motor and sensory axon guidance. While culture studies suggest that targets are also tropic for sympathetic axons (Chamley et al., 1973; Chamley and Dowel, 1975), it is unclear whether tropism plays a role in normal development.

To determine whether target tissues are required to guide sympathetic axons to their destination, we examined the development of innervation in footpads of tabby mice that lack sweat glands. Tabby $(\mathrm{Ta})$ is an X-linked mutation affecting morphogenesis of epidermal derivatives, including sweat glands. It delays eye opening and tooth eruption and completely prevents sweat gland development (Falconer, 1952; Gruneberg, 1971; Blecher, 1986). The tabby sequence is unknown but the gene is believed to play an important role in dermal induction of epidermal derivatives (Mayer and Green, 1978). Footpads of tabby mice lack sympathetic fibers with neurotransmitter properties characteristic of sweat gland innervation (Rao et al., 1994). In contrast, epidermis and blood vessels and their sensory and noradrenergic sympathetic innervation appear normal. Thus, the tabby mutation selectively removes one peripheral target tissue from footpads, leaving the remaining tissues and their innervation unaffected. It was unclear, however, whether sympathetic fibers that would have innervated glands failed to arrive in the glandless footpads or whether axons found their way to the target field and then were not maintained.

The postnatal development of sweat glands and their innervation is well characterized in rat. During the first week, catecholamine-containing unmyelinated sympathetic axons associate with gland primordia (Landis and Keefe, 1983). As the secretory coil is elaborated, the sympathetic plexus expands in concert. Concurrently, the plexus acquires ChAT, AChE, VIP, and calcitonin gene-related peptide (CGRP) and catecholamines decrease (Leblanc and Landis, 1986; Landis et al., 1988). Mouse sweat gland innervation is less well characterized. Adult gland innervation contains ChAT, AChE, and VIP (Rao et al., 1993; 1994). Since treatment of neonatal mice, like neonatal rats, with the noradrenergic neurotoxin 6-hydroxydopamine results in the absence of cholinergic sympathetic innervation of sweat glands in adult mice, mouse gland innervation also appears to undergo 
a developmental switch from noradrenergic to cholinergic (Yodlowski et al., 1984; Rao et al., 1994).

We found that sweat glands are not required for sympathetic axons to pathfind accurately to the presumptive target field. Since gland targeted axons reach the appropriate footpad region in the absence of forming glands, we assessed the target dependence of subsequent morphogenesis. While the presumptive gland innervation lingers in the target area for approximately 2 weeks, it does not elaborate an extensive plexus nor undergo changes in neurotransmitter phenotype.

\section{Materials and Methods}

Animals. All mice were obtained from the Jackson Laboratory (Bar Harbor, ME). We outcrossed heterozygous tabhy (B6CBACa- $A^{\omega-j} / \mathrm{A}-\mathrm{Ta} /$ O) females $\left(T_{a} /+\right)$ with BL57/6J males and then intercrossed the progeny to obtain a line with relatively high fertility. Tabby is an X-linked mutation and we analyzed both homozygous mutant females $(T a / T a)$ and hemizygous mutant males $(T a / y)$. Littermate wild-type $(1 / t)$ mice served as controls. Examination of footpads from mutant and wild-type mice of the inbred tabby strain yielded similar results. In all cases, the two large interdigital hind footpads were examined. Footpads from twenty adult wild-type and tabby mice were examined as well as footpads from six wild-type and tabby mice at each developmental age.

Bright-field microscopy. Mice were killed by ether inhalation and perfused transcardially with $4 \%$ paraformaldehyde in $0.1 \mathrm{M}$ phosphate buffer, $\mathrm{pH} \mathrm{7.3,} \mathrm{for} 10 \mathrm{~min}$. The footpads were dissected and immersed in fixative containing $2 \%$ paraformaldehyde and $2 \%$ glutaraldehyde in $0.1 \mathrm{M}$ phosphate buffer for at least $1 \mathrm{~d}$ at $4^{\circ} \mathrm{C}$. The tissue was rinsed in phosphate-buffered saline (PBS), $\mathrm{pH} 7.3$, post fixed in $2 \% \mathrm{OsO}_{4}$, stained with uranyl acetate, rinsed, dehydrated, and embedded in EPON resin (Polysciences, Warrentown PA). One micrometer plastic sections were stained with alcoholic toluidine blue.

Histochemistry. Catecholamine-containing fibers were localized using glyoxylic acid (de La Torre, 1980). Mice were killed via ether inhalation and footpads were dissected and frozen in Tissue Tek (Fisher, Pittsburgh, PA). Ten micrometer cryostat sections were melted onto slides and dipped in a solution containing $1 \%$ glyoxylic acid (Sigma, St. Louis. $\mathrm{MO}$ ), $0.2 \mathrm{M}$ potassium phosphate and $0.2 \mathrm{M}$ sucrose, $\mathrm{pH} 7.4$. The sections were dried in an air stream, heated to $95^{\circ} \mathrm{C}$ under mineral oil for $2.5 \mathrm{~min}$, and then coverslipped.

Acetylcholinesterase activity was localized using a modification of the Karnovsky-Roots procedure (Landis and Keefe, 1983). Ten micrometer sections of fresh tissue were melted onto slides and fixed in $4 \%$

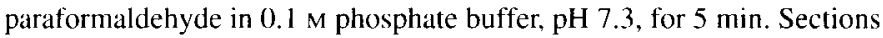
were rinsed with PBS and then incubated in a solution containing $2 \mathrm{~mm}$ acetylthiocholine iodide (Sigma), $66 \mathrm{~mm}$ maleic acid, $5 \mathrm{~mm}$ sodium citrate. $3 \mathrm{~mm}$ cupric sulfate, and $10 \mathrm{~mm}$ tetraisopropyl pyrophosphoramide (Sigma), which reduces nonspecific esterase activity. After 20 min, potassium ferricyanide was added (incubation solution concentration $5 \mathrm{~mm}$ ) and the reaction continued for $1.5 \mathrm{hr}$. Sections were rinsed in PBS and the reaction product intensified with methylene blue, which also serves as a counterstain. The sections were then dehydrated and coverslipped with Permount.

Immunohistochemistry. Mice were killed by ether inhalation and perfused with $4 \%$ paraformaldehyde for $10 \mathrm{~min}$. Footpads were dissected and immersed in fixative for an additional hour at room temperature, rinsed in PBS, then equilibrated in $30 \%$ sucrose in $0.1 \mathrm{M}$ phosphate buffer at $4^{\circ} \mathrm{C}$. Ten micron cryostat sections were dried onto gelatin coated slides and rinsed in PBS. The sections were incubated for $1 \mathrm{hr}$ at room temperature in dilution buffer containing $2 \%$ bovine serum albumin, $0.1 \%$ sodium azide, and $0.3 \%$ Triton X-100 in PBS. Sections were incubated with primary antiserum overnight, rinsed in PBS, and labeled with species specific secondary antisera conjugated to either fluorescein or rhodamine (Jackson Immunoresearch, West Grove, PA; Cappel, West Chester, PA) in dilution buffer with 5\% rat serum for 1 hr. The labeled sections were rinsed and cover slipped with $50 \%$ glycerol in PBS. All antisera were diluted 1:300. Some sections were double labeled for tyrosine hydroxylase and NPY or tyrosine hydroxylase and VIP by using anti-tyrosine hydroxylase generated in rabbit and antipeptide antisera generated in guinea pig. No secondary antiserum crossreactivity was observed in the double label procedure. The rabbit antiTH antiserum was purchased from Pel-Freez (Rogers, AR) and the CGRP antiserum from Amersham (Arlington Heights, IL). The guinea pig anti-VIP antiserum was generated against porcine VIP conjugated to PPD. Anti-NPY antiserum was generated in guinea pig against porcine NPY conjugated to keyhole limpet hemocyanin.

\section{Results}

Development of footpads in control and tably mulant mice

Most footpad development occurred postnatally. On the first postnatal day (PO), small buttons of raised epidermis were evident in control mice. These elevations developed into mature pads during the first postnatal month. At P0-P1, shallow epidermal folds began to give rise to the sweat glands by growing down from the epidermis into the footpad core. By $\mathrm{P} 4$, discrete primordia were evident (Fig. $1 a$ ). Between P7 to 10, the developing secretory tubules coiled to form glands (Fig. 1b). By P14, individual glands were surrounded by a connective tissue sheath. Secretory and myoepithelial cells could be distinguished in the secretory tubule and the lumen of each tubule was evident (Fig. 1c). At P7 and later, axon bundles containing sensory axons and associated Schwann cells were present in the connective tissue between the glands (Fig. $1 b, c$ ). In adult control footpads, sections through the gland region revealed portions of the secretory coil characterized by a lumen ringed by densely stained gland cells (Fig. 1d). Fascicles of axons, many of which were myelinated, were apparent in the center of the footpad. Numerous capillaries were present beneath the epidermis and interspersed among the secretory coils (Fig. $1 c, d$ ).

While the external appearance of the developing footpads of $t a b b y$ mutant mice superficially resembled that of wild type mice, the development of the internal tissue compartments differed. The basal margin of the epidermis of $t a b b y$ mutant footpads, like that of wild-type footpads, was infolded. Unlike the epidermal ridges which form gland primordia in wild-type mice. gland primordia do not form at the dermal/epidermal junction in footpads of tabby mutant mice (Fig. le). As the mutant mice matured, the pad continued to enlarge but gland secretory tubules were never detected (Fig. 1f). When compared to footpads of control mice, the epidermis appeared thicker, and the papillary layer and hypodennal dense connective tissue more extensive in footpads of tabby mice. At 7 and $14 \mathrm{~d}$, axon bundles containing sensory fibers were evident in the central region of the tabby footpad as in wild-type footpads (Fig. $1 f, g$ ). In adult tabby mutants, fascicles of myelinated sensory axons and small blood vessels were present in the subcutaneous tissue of the footpad (Fig. 1h).

\section{Development of footpad innervation in normal and tabby mutant footpads}

Approximately $2 \mathrm{~d}$ after birth, catecholamine-containing fibers appeared in peripheral nerves and associated with blood vessels at the base of the footpads of wild type mice. Between P4-P5, catecholaminergic fibers grew into the pad proper to associate with the gland primordia that had already grown into the central region of the pad (Fig. 2a). At the earliest stages of innervation, only a few, unbranched fibers were detected in the target field in each tissue section. From P7 to 14, a dense fluorescent plexus was elaborated around the developing glands (Fig. 2b). During the third postnatal week, although an extensive axonal plexus could be detected in association with glands, catecholamine fluorescence decreased in intensity and progressively fewer gland associated fibers were reactive (Fig. $2 c$ ). In contrast, throughout development the fluorescence intensity of fibers associated with the blood vessels at base of the pad remained robust. 

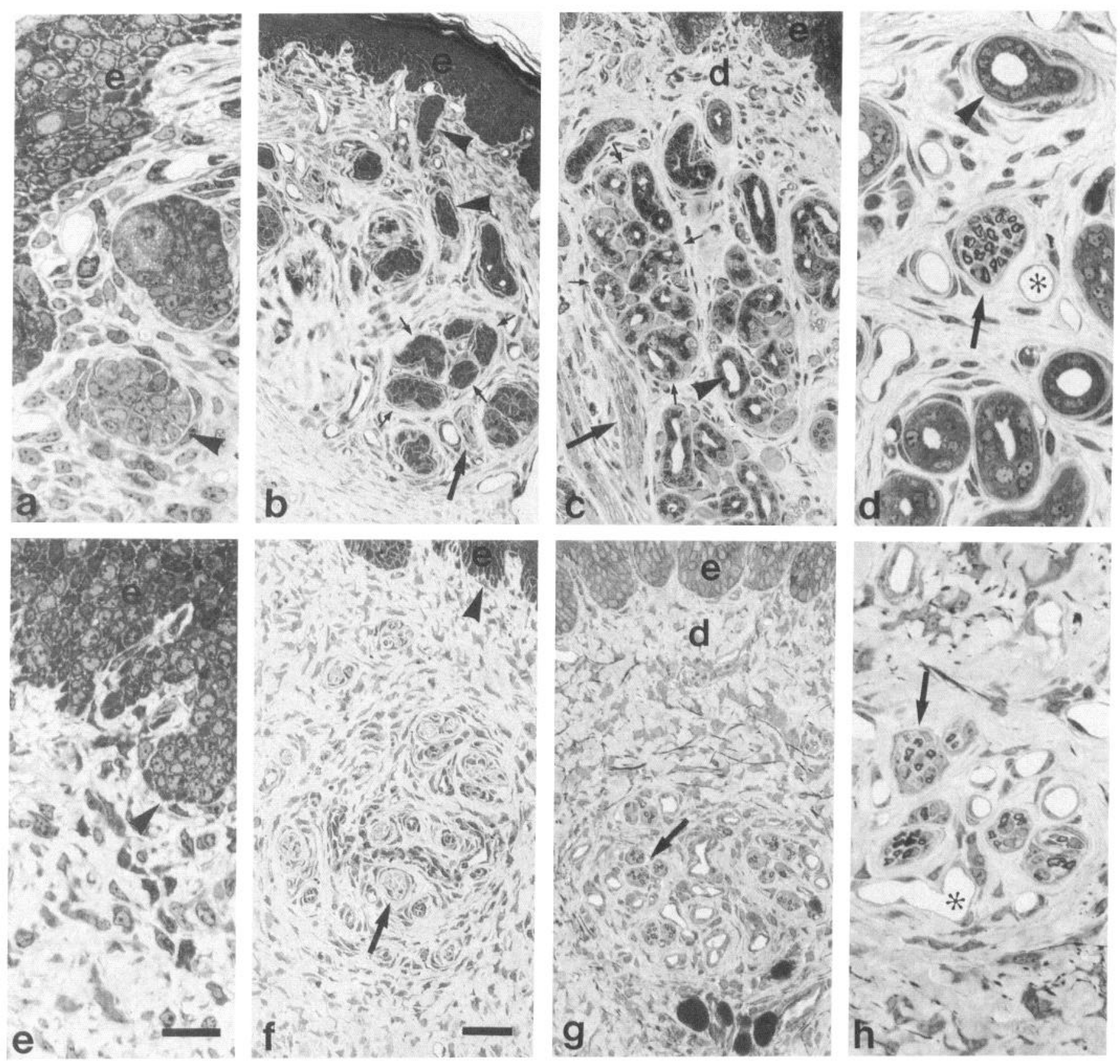

Figure 1. Footpad development in wild-type and tabby mutant mice. $a-d$ are photomicrographs from wild-type footpads while $e-h$ are from $t a b b y$ footpads. $a$, In P4 wild-type footpads, sweat gland primordia (arrowhead) have begun to extend from the epidermis toward the pad core. $e$, In contrast, although the epidermis of tabby mutant footpads, like that of wild type footpads, has infoldings (arrowhead), these do not extend into the underlying dermis or differentiate into gland tissue. $b$, At P7 in wild-type mice, the forming sweat glands, one of which is indicated by small arrows, occupy the central region of the footpad. The epidermal origin of a secretory tubule is indicated by an arrowhead. Bundles containing sensory axons (large arrow) are present between the forming glands. The sensory origin of these fibers is confirmed by their presence in sympathetomized animals. $(f)$ At P7, the footpads of tabby mutant mice are devoid of gland anlage. Axon bundles (arrow) are seen in the central region of the pad. The epidermis is folded as in wild-type footpads (arrowhead). $c$, By P14, the secretory tubules of wild-type mice are tightly coiled and the lumen of each is patent (arrowhead). One gland is outlined by small arrows. Bundles of myelinated sensory axons are evident, adjacent to the glands (arrow). Dense connective tissue, $d$, separates the epidermis from the subcutaneous tissue of the pad core. $g$, Although the footpads of tabby mutant mice are similar in size to those of wild-type mice at P14, they lack glands. The arrow indicates a bundle of myelinated sensory axons. Since the footpads are devoid of glands, the axon bundles are more obvious than in control footpads. Dense connective tissue, $d$, separates the epidermis from the subcutaneous tissue of the pad core. $d$, At higher magnification, gland tubules (arrowhead) and small blood vessels (asterisk) are evident in wild-type pads at P17. A fascicle containing myelinated and umyelinated sensory axons is indicated by the arrow. $h$, Tabby mutant pads at P17 also contain fascicles with myelinated axons (arrow). Small blood vessels are present (asterisk) while gland tubules are not absent. All figures are $1 \mu \mathrm{m}$ plastic sections stained with toluidine blue. In all figures, $e$ indicates the epidermis. Scale bar in $e, 20 \mu \mathrm{m} ; a, d, e$, and $h$ are the same magnification. Scale bar in $f, 40 \mu \mathrm{m} ; b, c, f$, and $g$ are the same magnification. 

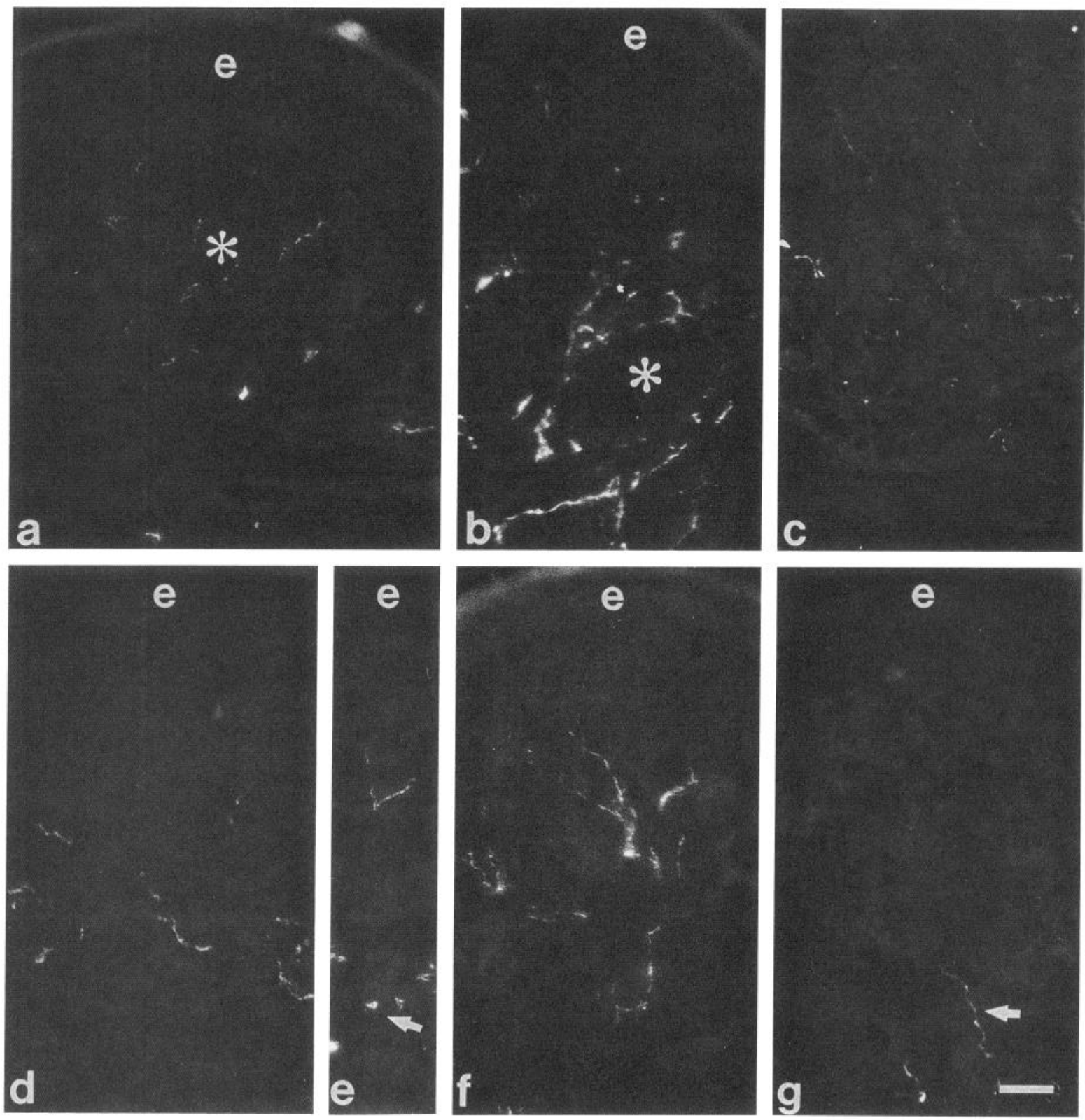

Figure 2. Development of catecholamine histofluorescence. All figures are oriented as in Figure 1 so that the epidermis is at the top of the micrograph and the base of the footpad is at the bottom of the micrograph. $a$, In control footpads at P4, fluorescent fibers are present around the gland anlage (asterisk). $b$, At P10, brightly fluorescent fibers are associated with the forming secretory coils (asterisk). $c$, At P14, catecholamine fluorescence is significantly decreased in fibers associated with the glands. The epidermis is not included in this micrograph. $d$ and $e$, In footpads of P4 tabby mutant mice, catecholamine fluorescent fibers are present in bundles at the base of the footpad (arrow) and extend up into the central core of the pad. $f$, At P10, a sparse plexus of catecholaminergic fibers is present in the region of the footpad in which glands should have developed. $g$, At P14, virtually all catecholamine fluorescence has disappeared and only faint, usually solitary fibers are seen at the base of the footpad (arrow). Glyoxylic acid induced fluorescence was visualized in $10 \mu \mathrm{m}$ frozen sections. In all figures, $e$ indicates the epidermis. Scale bar in $g, 40 \mu \mathrm{m}$; all micrographs are the same magnification.

Catecholamine-containing fibers also appeared in the footpads of tabby mutant mice around postnatal day 2. As in wild-type mice, the fluorescent fibers were present in peripheral nerves and associated with large blood vessels at the base of the pad. Between P4 and 5, catecholaminergic fibers appeared in the region of the footpad that in normal mice is occupied by developing sweat glands (Fig. $2 d$ ). The density of innervation was similar to that in control mice when the glands and fibers initially come in contact. Typically, a few moderately branched fibers were detected in each section of the central aspect of the pad (Fig. $2 d, e$ ). Between 4 and $14 \mathrm{~d}$, with only rare exceptions, these fibers were restricted to the core of the pad: despite the absence of their normal target tissue, they did not innervate the most distal portions of the pad at the junction between the dermis and epidermis nor were any seen to penetrate into the epidermis (Fig. $2 d-f$ ). The catecholamine-containing fibers did not branch extensively and the plexus that they formed was sparse in comparison to the rapidly expanding plexus of normal mice (Fig. 2f). At P14, only solitary fluorescent fibers were seen (Fig. $2 g$ ) and by P21, fluorescent fibers were not detected in the pre- 
sumptive gland region. As in wild-lype mice, catecholamine histofluorescence was robust in the innervation of the blood vessels subjacent to the gland target field throughout development.

The development of the sympathetic footpad innervation was also assayed by immunocytochemical localization of tyrosine hydroxylase, the rate-limiting enzyme in catecholamine synthesis. The initial development of tyrosine hydroxylase immunoreactivity paralleled that of catecholamine histofluorescence for both wild-type and tabby mutant mice. At P1-2, in both tabby and wild-type mice,immunoreactive axons were present in the base of the footpad in nerve bundles and associated with arteries and arterioles in the central region of footpad. The blood vessel innervation sometimes contained immunoreactivity for neuropeptide Y (NPY). In control mice, by P4-5 tyrosine hydroxylase immunoreactive fibers were associated with the presumptive sweat glands in the central region of the pad. During the next 2 weeks, this plexus increased in density and immunofluorescence intensity as the glands and their innervation matured (Fig. 3a,c). In contrast to sympathetic axons associated with blood vessels, those that innervate the developing glands always lacked detectable NPY immunoreactivity (Fig. 4a,b). While catecholamine fluorescence disappeared from the gland innervation by P21, there was little or no diminution in the intensity of tyrosine hydroxylase immunoreactivity.Expression of tyrosine hydroxylase immunoreactivity in the absence of catecholamines has been reported in a variety of other neurons in both the peripheral and central nervous system (Landis et al., 1987; Kitahama et al., 1990; Nagatsu et al., 1990; see discussion in Rao et al., 1994).

From P2 to P14, the footpads of tabby mutant mice also contained tyrosine hydroxylase immunoreactive, NPY-negative fibers (Figs. $3 e, g, 4 c, d$ ). Many of these fibers were not associated with blood vessels but were typically observed in the central portion of the pad. Tyrosine hydroxylase containing fibers were detected very infrequently near the dermal/epidermal boundary. During this period, the TH positive innervation of the presumptive gland target region in tabby mutant footpads was very sparse, resembling that of control footpads just as the gland primordia have begun to invade the central region of the footpad. Near the end of the second postnatal week, the nonvascular tyrosine hydroxylase immunoreactivity had largely disappeared while the tyrosine hydroxylase and NPY immunoreactive fibers innervating the vasculature remained. Since tyrosine hydroxylase immunoreactivity is normally maintained by the adult sweat gland innervation in mice, the disappearance of tyrosine hydoxylase from the footpads of tabby mice provides evidence that the gland-targeted sympathetic fibers have been lost.

During development, the sympathetic innervation of sweat glands, but not of blood vessels, acquires VIP immunoreactivity and acctylcholincstcrase activity. During the sccond postnatal week, the gland innervation of wild-type mice became VIP immunoreactive (Fig. 3b,d). In double label studies, the distribution of the VIP-immunoreactive fibers overlapped with that of tyrosine hydroxylase immunoreactive fibers. The intensity of VIP staining in the sweat gland innervation increased during the third postnatal week. In contrast, sympathetic fibers innervating bluod vessels in the pad were never VIP immunoreactive. The gland associated sympathetic innervation also developed $\mathrm{AChE}$ activity. During the second postnatal week, AChE positive fibers became evident between the coils of the gland secretory tubule (Fig. 5). In marked contrast to the acquisition of VIP immunoreactivity and $\mathrm{AChE}$ activity by the developing sweat gland innervation of wild-type mice, no VIP immunoreactive or $\mathrm{AChE}$ positive fibers were detected in the footpads of developing labby mutant mice. (Figs. $3 f, h, 5$ ).

In addition to sympathetic innervation, mouse footpads are also innervated by sensory fibers some of which are immunoreactive for CGRP (Rao et al., 1994). In both tabby mutant and control mice, a few CGRP containing fibers were typically present in footpads at P1. During the first two postnatal weeks, the extent of sensory innervation increases significantly. At P17 in control mice, the CGRP fibers were seen extending through the gland field and innervating the dermal/epidermal junction around the periphery of the footpad (Fig. 6a). Fine, individual fibers extended deep into the epidermal folds where the staining often appeared punctate. In footpads of tabby mice, CGRP fibers were also seen traversing the central region of the footpad and extending to the epidermal folds at the pad margin at P17 (Fig. $6 b)$. As in control mice, immunoreactive fascicles in the pad core were thick, while distal fibers associated with the skin were more delicate and appeared beaded. In both control and tabby mutant footpads, the bundles of sensory fibers revealed by CGRP immunoreactivity correspond to the axon fascicles evident in plastic sections (Fig. 1). The presence of sensory axons, some of which are visualized as CGRP-immunoreactive fibers and axon fascicles in plastic sections, precluded the use of a pan-axonal marker to identify sympathetic fibers in adult $t a b b y$ footpads.

\section{Discussion}

Analysis of tabby footpads reveals that sympathetic axons transiently innervate the area in which sweat glands would have formed. The nonvascular catecholaminergic innervation of footpads is initially sparse in both wild-type and tabby footpads. Normally, the gland-associated fibers ramify extensively in conjunction with sweat gland morphogenesis and undergo a switch in transmitter phenotype. While sympathetic fibers are present in the presumptive gland target field in tabby footpads during the first two postnatal weeks, the axonal plexus fails to expand and the axons retain catecholamines and do not acquire $\mathrm{AChE}$ or VIP. Since tyrosine hydroxylase immunoreactivity and catecholamines remain in association with blood vessels, tabby does not affect sympathetic neurons directly.

Our observations indicate that developing sweat gland innervation is not dependent upon the presence of gland primordia to navigate precisely to its target field. In control mice, nascent glands begin to extend into the footpad core to form the target field as sympathetic axons arrive at the footpad base. In tabby, sympathetic axons invade the central region of the pad despite the absence of developing glands. How sympathetic axons destined to innervate sweat glands pathfind to the footpad and then invade it is unclcar. Since sensory axons precede sympathetic axons to the footpad (unpublished observations), sympathetic axons may follow them in peripheral nerves as muscle sensory axons have been proposed to follow motoneuron axons (Honig et al., 1986; Landmesser and Honig, 1986). Once sympathetic axons arrive at the footpad base, however, they follow a trajectory distinct from that of sensory axons, stopping mid-pad rather llan projecting to the dermal/epidermal border.

Despite the absence of their normal target tissue, gland-targeted sympathetic axons do not invade the target fields of sensory fibers at the footpad margin nor do they associate with blood vessel targets of noradrenergic sympathetic fibers. In adult rodents, substance $\mathrm{P}$ and CGRP-immunoreactive sensory axons form a plexus at the boundary between the epidermis and dermis 

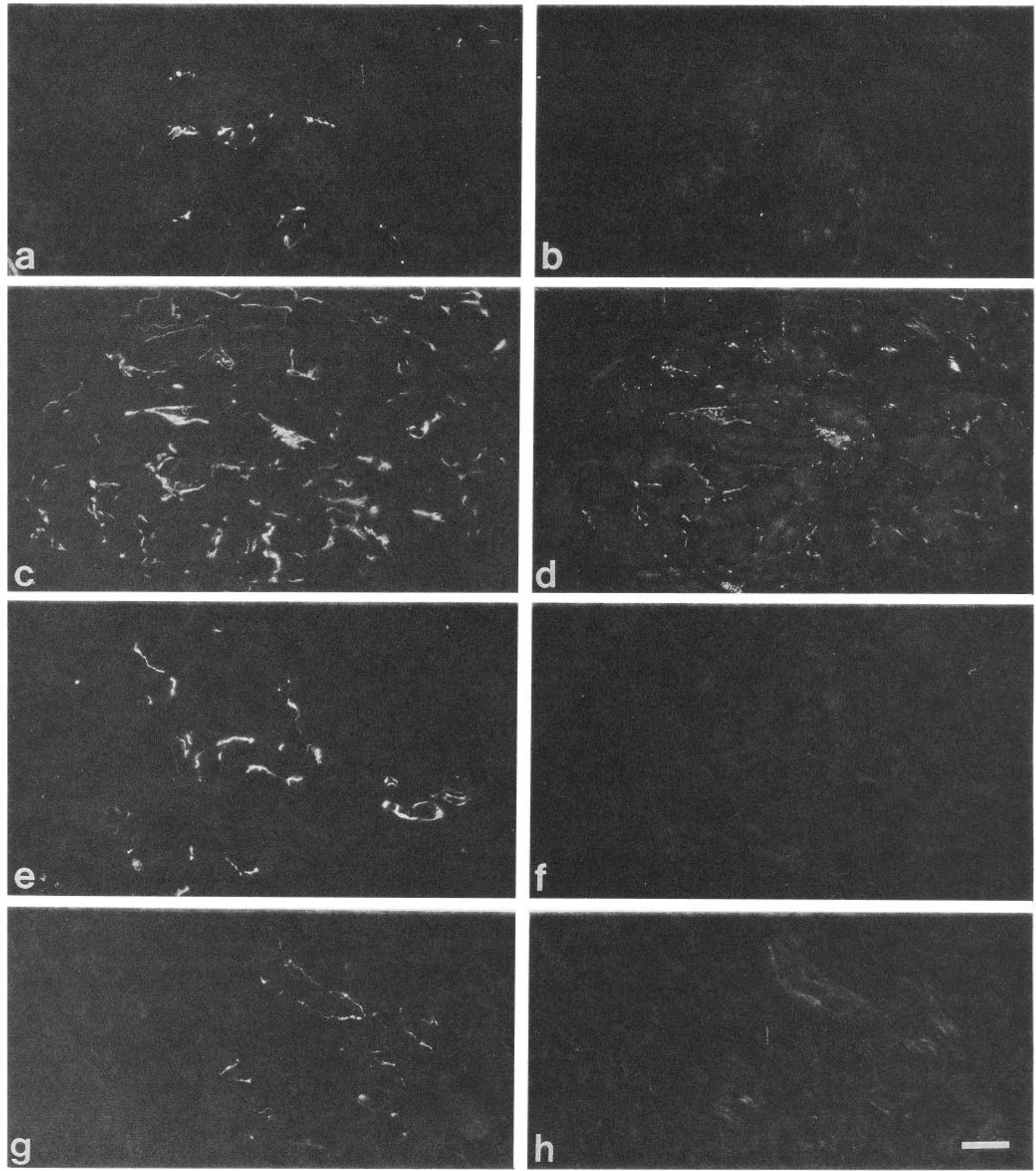

Figure 3. Colocalization of tyrosine hydroxylase and VIP immunoreactivity. $a$, At P7, the developing innervation of sweat glands in the footpads of wild-type mice contains immunoreactivity for tyrosine hydroxylase. $b$, VIP immunoreactivity is undetectable in the same section. $c$, At P14, the sweat gland innervation in footpads of control mice is strongly immunoreactive for tyrosine hydroxylase, $d$, The same axons contain immunoreactivity for VIP. The VIP immunofluorescence appears more granular than that of tyrosine hydroxylase, presumably because the neuropeptide is present in dense core vesicles while the synthetic enzyme is cytoplasmic. $e$, At P7, the presumptivc gland region of tabby mutant mouse footpads contains tryosine hydroxylase immunoreactive fibers. $f$. No VIP immunoreactivity is detectable. $g$. At P14, tyrosine hydroxylase immunoreactive fibers in the footpads of tabby mutant mice are reduced in number and in fluorescence intensity. $h$, In contrast to the tyrosine hydroxylase fibers innervating sweat glands in the footpads of control mice, those in the footpads of tabby mutant mice do no contain detectable VIP. Ten micrometer frozen sections through the central region of the footpad were stained with rabbit anti-tyrosine hydroxylase and guinea pig anti-VIP and species specific secondary antisera conjugated to different fluorochromes. Scale bar in $h, 40 \mu \mathrm{m}$; all photomicrographs are the same magnification. 

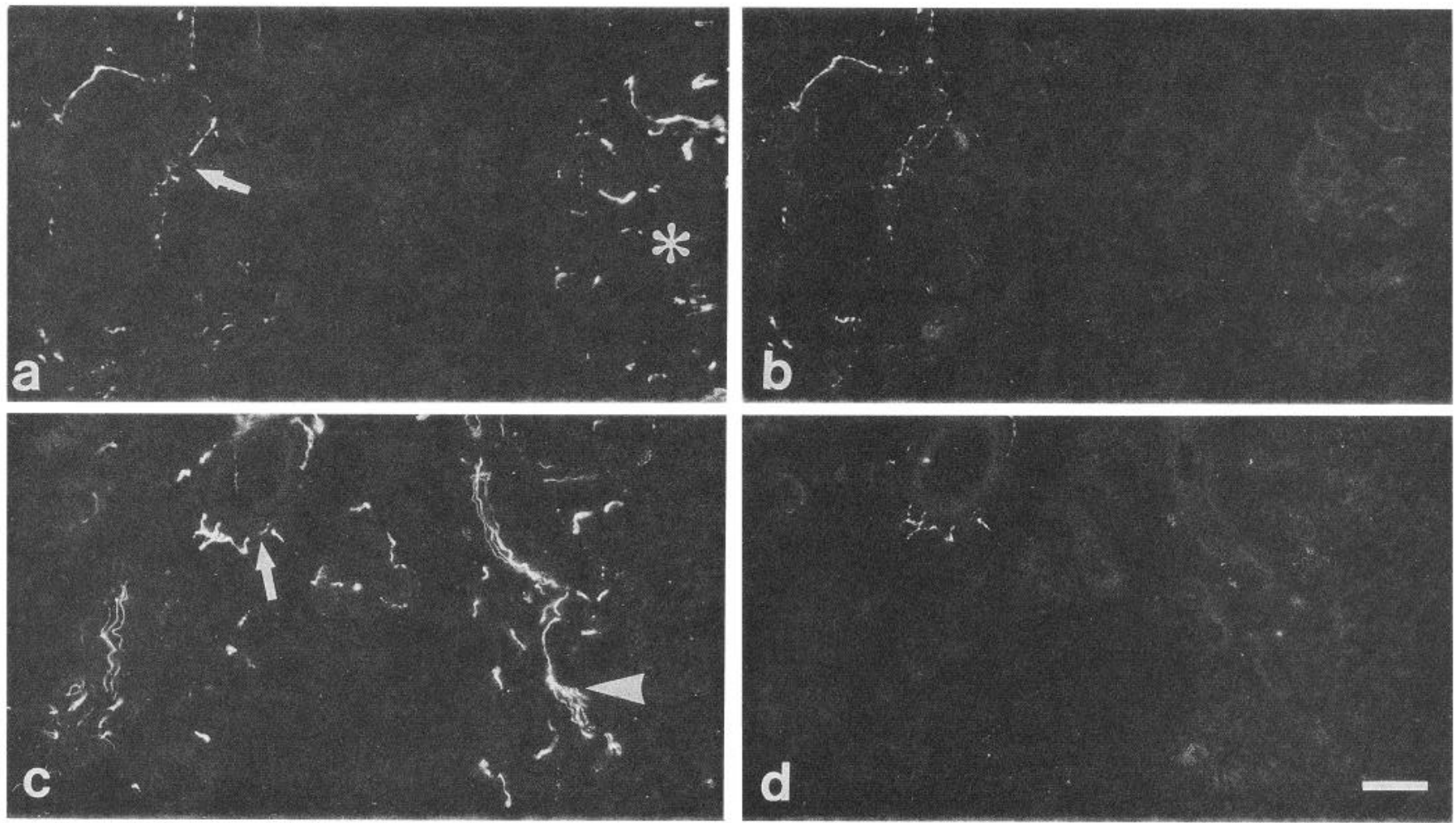

Figure 4. Colocalization of tyrosine hydroxylase and NPY immunoreactivity. In these micrographs, the base of the footpad is to the left and the central region is to the right. The epidermis is not included. $a$, At P14 in control mouse footpads, tyrosine hydroxylase immunoreactive fibers are associated with both sweat glands (asterisk) and blood vessels (arrow). $b$, The blood vessel sympathetic innervation contains NPY immunoreactivity while the sweat gland innervation does not. $c$, In footpads of P14 tabby mutant mice, tyrosine hydroxylase immunoreactive axons are associated with blood vessels (arrow) and are present in nerve bundles at the base of the footpad (arrowhead). $d$, As in control mice, the blood vessel innervation in tabby footpads contains immunoreactivity for NPY. Sympathetic axons in the region where glands would normally form lack NPY immunoreactivity. This particular field has an unusually large number tyrosine hydroxylase immunoreactive fibers remaining. Ten micrometer frozen sections were stained with rabbit anti-tyrosine hydroxylase and guinea pig anti-NPY and species specific secondary antisera conjugated to different fluorochromes. Scale bar in $d, 40 \mu \mathrm{m}$; all micrographs are the same magnification.
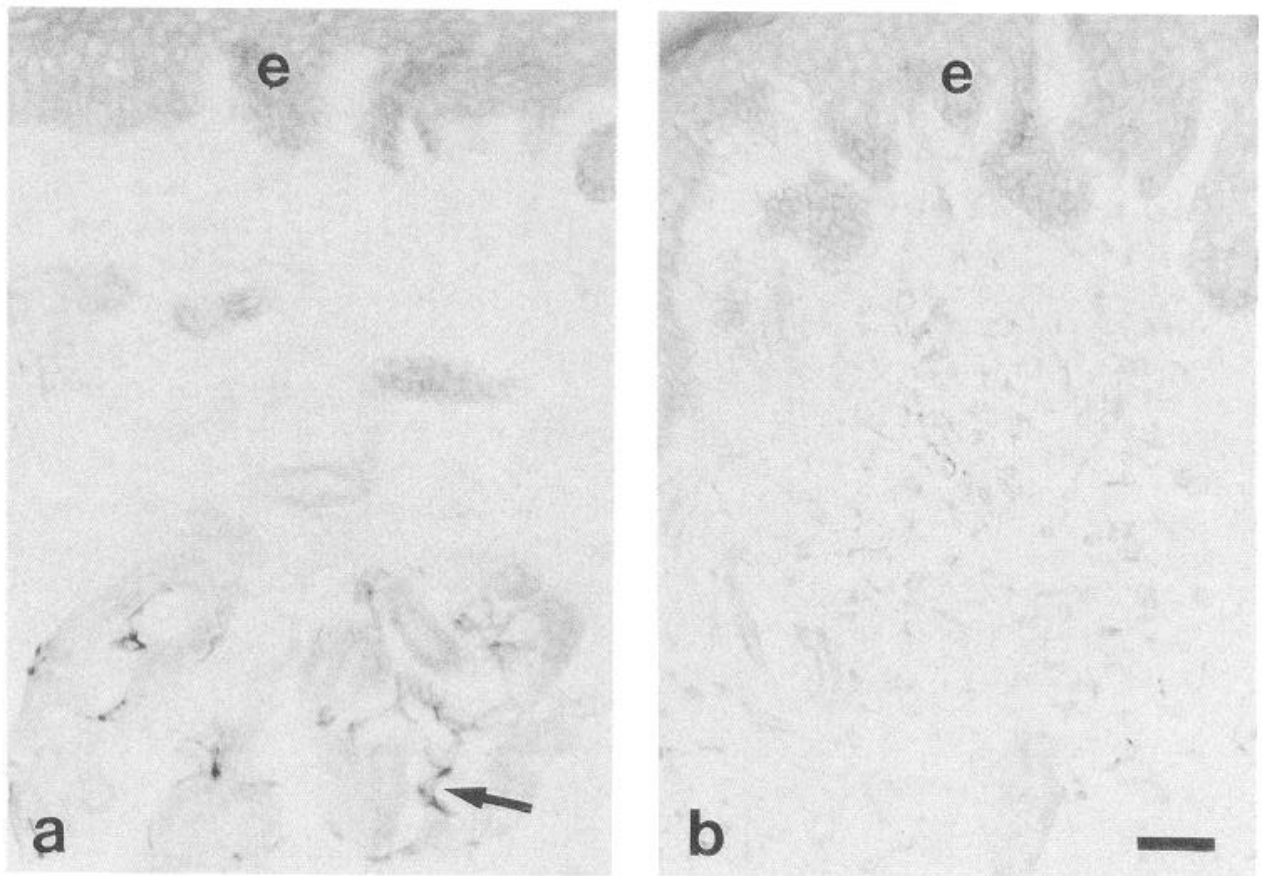

Figure 5. Development of AChE. The figures are oriented as in Figure 1 so that the epidermis is at the top of the micrograph and the base of the footpad is at the bottom of the micrograph $a$, At P14 the footpads of wild-type mice possess darkly stained AChE positive fibers (arrow) interdigitated with the developing secretory coils. $b$, At P14, no AChE positive fibers are evident in the footpads of tabby mutant mice. The discontinuous speckles evident in this photomicrograph are processes of melanocytes present in the dermis. Ten micrometer frozen sections were reacted for $\mathrm{AChE}$ and the reaction product intensified with methylene blue which also lightly counterstains the epidermis and sweat glands. In both micrographs, $e$ indicates the epidermis. Scale bar in $b, 40 \mu \mathrm{m}$; all micrographs are the same magnification 
Figure 6. CGRP immunoreactivity in sensory innervation in footpads. The figures are oriented as in Figure 1 so that the epidermis is at the top of the micrograph and the base of the footpad is at the bottom of the micrograph. $a$, CGRP immunoreactive sensory fibers are present in the footpads of control mice at P17. Thick fascicles containing CGRP-immunoreactive fibers are present in the base of the footpad (arrow). Asterisk indicates gland region. Fibers associated with the dermis/epidermis junction are fine and punctate (arrowhead). $b$, At P17 in tabby mutants, large CGRP-immunoreactive fascicles traverse the presumptive sweat gland target field (arrow). Very fine, often punctate fibers are associated with the epidermal folds at the margin of the pad (arrowhead). In both micrograpphs, $e$ indicates the epidermis. Scale bar in $b$, $40 \mu \mathrm{m}$; both micrographs are the same magnification.
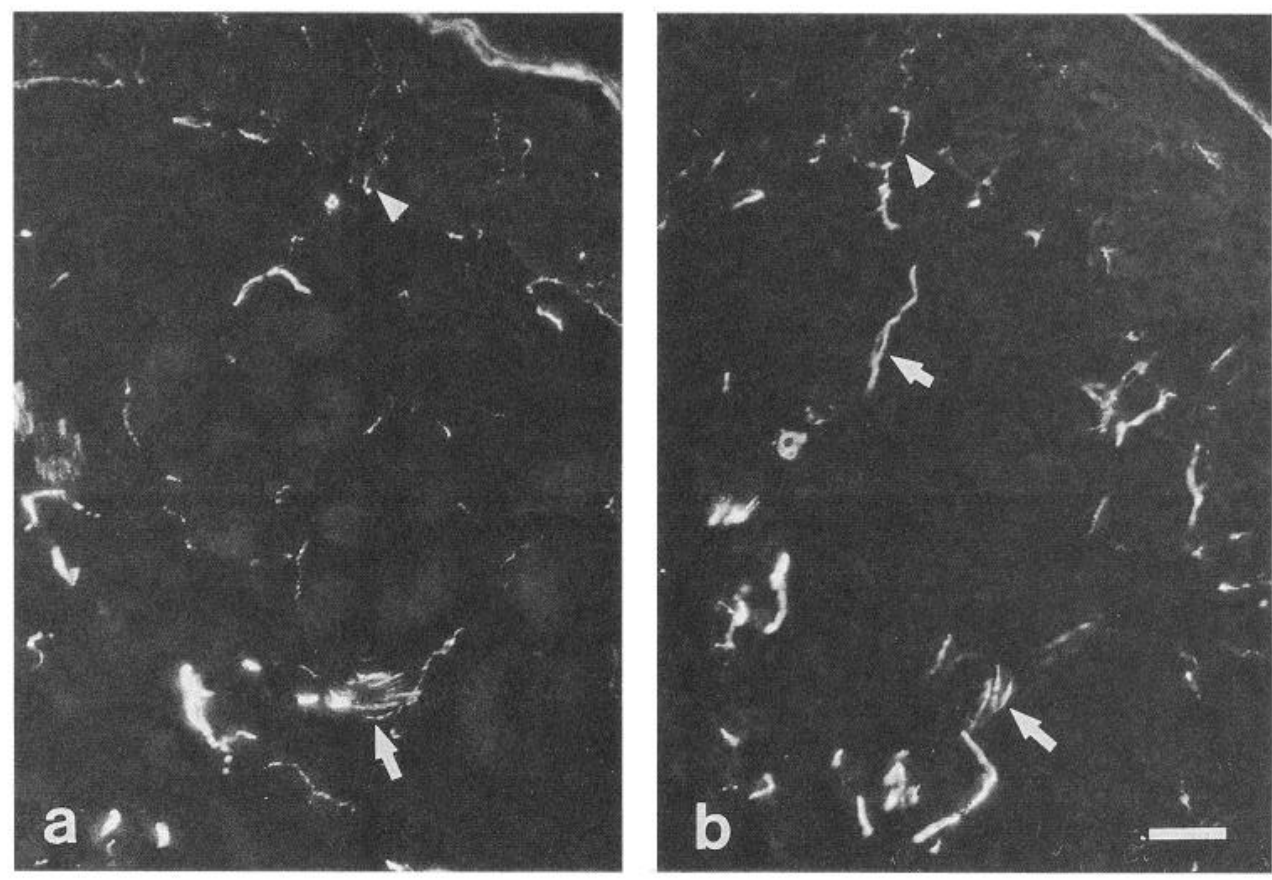

(Kruger et al., 1989; Rao et al., 1994) and sensory terminals are present in this region before sympathetic axons invade the pad (unpublished observations). Since the epidermis and dermis of other skin regions contain NGF shortly after sensory axons reach them (Davies et al., 1987; Schecterson and Bothwell, 1992), it seems likely that early postnatal footpad skin also contains NGF. Therefore while one might have expected that gland-targeted sympathetic axons would continue to grow toward the epidermis when they fail to encounter their normal target tissue, only very rarely did they. Insufficient NGF may be present in skin to attract sympathetic axons due to removal by earlier arriving sensory neurons. Sympathetic fibers do invade the footpad margin, however, when epidermal NGF overexpression is driven by a keratin promoter (Guidry et al., 1994). Thus, there may be a threshold for an NGF tropic effect. Two factors in addition to NGF could contribute to such a threshold. First, the dense connective tissue immediately distal to the region where glands form may be a barrier to sympathetic axon growth. Second, the dermal-epidermal junction may not support sympathetic axons which penetrate the dense connective tissue. Consistent with this possibility, cultured epidermal cells cause sympathetic, but not sensory, growth cones to collapse (Vos et al., 1991). Since the catecholaminergic sympathetic plexus around blood vessels in tabby footpads is normal, gland-targeted sympathetic axons do not appear to associate with vasculature. Thus, restriction of sympathetic fibers to their appropriate domain, glands or vasculature, is finely regulated.

Although sympathetic fibers pathfind to the region of tabby footpads where glands normally develop, shortly after arrival the axonal arborization in mutant footpads becomes distinguishable from control. Normally, innervation density increases rapidly as gland primordia form secretory coils. Plexus expansion occurs even though sympathetic terminals never contact gland cells directly (Landis and Keefe, 1983), suggesting that diffusible trophic substances are involved. NGF is a candidate for plexus induction. The developing sweat gland innervation is NGF dependent (Landis et al., 1985; Hill et al., 1988), and in other tissues, sympathetic innervation density is correlated with NGF mRNA and protein (Korsching and Thoenen, 1983; Shelton and Reichardt, 1984). Several lines of evidence suggest that NGF influences sympathetic axon branching. In culture, local NGF concentrations influence neurite growth (Campenot, 1977). The increased number of sympathetic axons in nerves of transgenic mice in which NGF expression is driven by the dopamine- $\beta$ hydroxylase promoter has been attributed to branching elicited by high local NGF concentrations (Hoyle et al., 1993). NGF ectopically produced by pancreatic beta cells under control of the insulin promoter significantly increases sympathetic innervation of islets (Edwards et al., 1989) and NGF overexpressed under the control of a keratin promoter increases sympathetic innervation of hairy skin (Albers et al., 1994).

The fate of the gland-targeted innervation in tabby mice is unclear. Since tyrosine hydroxylase is maintained by mouse sweat gland innervation after catecholamines disappear, it is a marker for sympathetic gland innervation in adulthood. In contrast to wild-type mice, tyrosine hydroxylase disappears from tabby footpads by P20. While it seems most likely that without target-derived trophic factor the neurons of origin degenerate, we can not exclude the possibility that the axons have withdrawn from the footpad and/or established collaterals outside the footpads which sustain them. It is surprising that axons remain in the presumptive target area for so long, given the absence of their normal target and the failure of sympathetic axons to associate with the epidermis. In the early postnatal period, sympathetic neurons require NGF for survival. Anti-NGF treatment causes the death of virtually all sympathetic neurons (Levi-Montalcini and Booker, 1960; Gorin and Johnson, 1979, 1980). Cultured neonatal sympathetic neurons die within $24 \mathrm{hr}$ without exogenous NGF (Chun and Patterson, 1977) and neonatal neurons grown for 1 week with exogenous NGF die within 48 hours of NGF withdrawal (Martin et al., 1988). In contrast, sympathetic axons remain in tabby footpads for almost 2 weeks, suggesting they are trophic factor independent for a longer period 
of time than previously thought or there is an alternative local source that eventually proves insufficient.

The observation that sympathetic axons in tabby footpads do not alter their transmitter properties before they disappear is consistent with previous findings suggesting that sweat glands provide a retrograde instructive signal (Schotzinger and Landis, $1988,1990)$. When sweat gland primorida are replaced with parotid gland, a noradrenergic target, the presumptive sweat gland innervation remains catecholaminergic. Evidence for an inductive role of sweat glands was obtained when gland primordia transplanted to hairy skin caused noradrenergic sympathetic in nervation to lose catecholamines and become cholinergic and peptidergic. We could not exclude the possibility that parotid gland inhibited an intrinsically regulated transmitter switch. In the present studies, we found that sympathetic axons containing catecholamines and lacking AChE and VIP are still present in tabby footpads between P10 and P14, when catecholamines decline and AChE and VIP appear in wild-type footpads. Since ChAT activity is barely detectable in wild-type mouse footpads at P12 when sympathetic axons remain in tabby footpads, we could not determine whether the induction of this property was also affected. Thus, the gland-targeted innervation does not appear to alter most, and possibly all, its neurotransmitter properties in the absence of sweat glands. Since the remaining footpad tissues appear normal, these findings identify sweat glands as the source for the differentiation factor in footpads, consistent with reduced cholinergic inducing activity in tabby footpads and the production of cholinergic differentiation activity by cultured sweat gland cells (Habecker and Landis, 1994; Habecker et al., 1995).

Many aspects of sympathetic neuron development are dependent upon interactions with target tissues. As summarized above, sympathetic neuron survival requires target-derived trophic factors (Thoenen and Barde, 1980; Levi-Montalcini, 1987). Cell body and dendritic arbor size is influenced by the size of the target field (Voyvodic, 1987, 1989) and elaboration of an axonal plexus requires the presence of a target. Finally, the complement of neurotransmitters and neuropeptides expressed by sympathetic neurons is determined by the target tissues they innervate (Schotzinger and Landis, 1988, 1990; Tyrrell and Landis, 1994). The present finding that sympathetic axons pathfind accurately to the target field in the absence of a target provides a striking example of target-independent development.

\section{References}

Albers K, Wright D, Davis B (1994) Over-expression of NGF in the epidermis of transgenic mice causes hypertrophy of the peripheral nervous system. J Neurosci 14:1422-1432.

Blecher S (1986) Anhidrosis and absence of sweat glands in mice hemizygous for the Tabby gene: supportive evidence for the hypothesis of homology between Tabby and human anhidrotic (hypohidrotic) ectodermal dysplasia. J Invest Dermatol 87:720-722.

Bovolenta P, Dodd J (1991) Perturbation of neuronal differentiation and axon guidance in the spinal cord of mouse embryos lacking a floor plate: analysis of Danforth's short tail mutation. Development 113:625-639.

Campenot RB (1977) Local control of neurite development by nerve growth factor. Proc Natl Acad Sci USA 74:4516-4519.

Chamley JH, Dowel JJ (1975) Specificity of nerve fibre 'attraction' to autonomic effector organs in tissue culture. Exp Cell Res 90:1-7.

Chamley JH, Goller I, Burnstock G (1973) Selective growth of sympathetic nerve fibers to explants of normally densely innervated autonomic effector organs in tissue culture. Dev Biol 31:362-379.

Chun LLY, Patterson PH (1977) Role of nerve growth factor in the development of rat sympathetic neurons in vitro. I. Survival, growth and differentiation of catecholamine production. J Cell Biol 75:694704.

Davies AM, Bandtlow C, Heumann R, Korsching S (1987) Timing and site of nerve growth factor synthesis in developing skin in relation to innervation and expression. Nature 326:353-358.

de La Torre JC (1980) An improved approach to histofluorescence using the SPG method for tissue monoamines. J Neurosci Methods $3: 1-5$.

Edwards RH, Rutter W, Hanahan O (1989) Directed expression of NGF to pancreatic beta cells in transgenic mice leads to selective hyperinnervation of the islets. Cell 58:161-170.

Falconer DS (1952) A totally sex-linked gene in the house mouse. Nature 169:664-665.

Goodman CS, Shatz CJ (1993) Developmental mechanisms that generate precise patterns of neuronal connectivity. Neuron 10(Suppl):77 90

Gorin PD, Johnson EM (1979) Experimental autoimmune model of nerve growth factor deprivation: effects on developing peripheral sympathetic and sensory neurons. Proc Natl Acad Sci USA 76:53825386.

Gorin PD, Johnson EM (1980) Effects of exposure to nerve growth factor antibodies on the developing nervous system of the rat: an experimental autoimmune approach. Dev Biol 80:313-323.

Gruneberg H (1971) The glandular aspects of the tabby syndrome in the mouse. J Embryol Exp Morphol 25:1-19.

Guidry G, Davis BM, Landis SC, Albers KM (1994) NGF expression in epidermis disrupts sympathetic innervation of sweat glands and differentially alters transmitter properties. Soc Neurosci Abstr 20: 451.4

Habecker BA, Landis SC (1994) Noradrenergic regulation of cholinergic differentiation. Science 264:1602-1604.

Habecker BA, Iresser SJ, Kao MS, Landis SC (1995) Production of sweat gland cholinergic differentiation factor depends on innervation. Dev Biol 167:307-316.

Heffner CD, Lumsden AGS, O'Leary DDM (1990) Target control of collateral extension and directional axon growth in the mammalian brain. Science 247:217-220

Hill CE, Jelinek H, Hendry IA, McLennan IS, Rush RA (1988) Destruction by anti-NGF of autonomic, sudomotor neurons and subsequent hyperinnervation of the foot pad by sensory fibers. J Neurosei Res 19:474-482.

Honig MG, Lance-Jones C, Landmesser L (1986) The development of sensory projection patterns in embryonic chick hindlimb under experimental conditions. Dev Biol 118:532-548.

Hoyle GW, Mercer EH, Palmiter RD, Brinster RL (1993) Expression of NGF in sympathetic neurons leads to excessive axon outgrowth from ganglia but decreased terminal innervation within tissues. Neuron 10:1019-1034.

Kitahama K, Geffard M, Okamura H, Nagatsu I, Mons N, Jouvet M (1990) Dopamine- and DOPA-immunoreactive neurons in the cat forebrain with reference to tyrosine hydroxylase-immunocytochemistry. Brain Res 518:83-94.

Korsching S, Thoenen H (1983) Nerve growth factor in sympathetic ganglia and corresponding target organs of the rat: correlation with density of sympathetic innervation. Proc Natl Acad Sci USA 80: 3513-3516.

Kruger L, Silverman JD, Mantyyyh PW, Sternini CW, Brecha NC (1989) Peripheral patterns of calcitonin gene-related peptide general somatic sensory innervation: cutaneous and deep terminations. J Comp Neur 280:2911-302.

Landis SC, Keefe D (1983) Evidence for neurotransmitter plasticity in vivo: developmental changes in the properties of cholinergic sympathetic neurons. Dev Biol 98:349-372.

Landis SC, Fredieu JR, Yodlowski M (1985) Neonatal treatment with nerve growth factor antiserum eliminates cholinergic sympathetic innervation of rat sweat glands. Dev Biol 112:222-229.

Landis SC, Jackson PC, Fredieu JR, Thibault J (1987) Catecholaminergic properties of cholinergic neurons and synapses in adult rat ciliary ganglion. J Neurosei 7:3574-3587.

Landis SC, Schwab M, Siegel RE (1988) Evidence for neurotransmitter plasticity in vivo. II. Immunocytochemical studies of rat sweat gland innervation during development. Dev Biol 126:129-138.

I andmesser I. (1984) The development of specific motor pathways in the chick embryo. Trends Neurosci 7:337-339.

Landmesser L, Honig MG (1986) Altered sensory projections in the 
chick hind limb following early removal of motoneurons. Dev Biol 118:511-531.

Leblanc G, Landis SC (1986) Development of choline acetyltransferase activity in the cholinergic sympathetic innervation of sweat glands. J Neurosci 6:260-265.

Levi-Montalcini R (1987) The nerve growth factor: thirty-five years later. EMBO J 6:1145-1154.

Levi-Montalcini R, Booker B (1960) Destruction of the sympathetic ganglia in mammals by an antiserum to a nerve growth factor protein. Proc Natl Acad Sci 46:384-391.

Lewis J, Chevallier A, Kieny M, Wolpert L (1981) Muscle nerve branches do not develop in chick wings devoid of muscle. J Embryol Exp Morphol 64:211-232.

Lumsden AGS, Davies AM (1986) Chemotropic effect of specific target epithelium in the developing mammalian nervous system. Nature 323:538-539.

Martin DP, Schmidt RE, DiStefano PS, Lowry OH, Cater JG, Johnson EM (1988) Inhibitors of protein synthesis and RNA synthesis prevent neuronal death caused by nerve growth factor deprivation. J Cell Biol 106:829-844.

Mayer TC, Green MC (1978) The epidermis is the site of action of Tabby (Ta) in the mouse. Genetics $90: 125-131$.

McCaig CD (1986) Myoblasts and myoblast-conditioned medium attract the earliest spinal neurites from frog embryos. J Physiol (Lond) $375: 39-54$.

Nagatsu I, Komori K, Takcuchi T, Sakai M, Yamada K, Karasawa N (1990) Transient tyrosine hydroxylase-immunoreactive neurons in the region of the anterior olfactory nucleu of pre- and postnatal mice do not contain dopamine. Brain Res 511:55-62.

Okamoto H, Kuwada JY (1991a) Alteration of pectoral fin nerves following ablation of fin buds and by ectopic fin buds in the Japanese medaka fish. Dev Biol 146:62-71.

Okamoto H, Kuwada JY (1991b) Outgrowth by fin motor axons in wildtype and a finless mutant of the Japanese medaka fish. Dev Biol 146:49-61.

O'Leary DDM, Koester S (1993) Development of projection neuron types, axon pathways, and patterned connections of the mammalian cortex. Neuron 10:991-1006.

O'Leary DDM, Bicknese AR, DeCarlos JA, Heffner CD, Koester SE, Kutja LJ, Terashima $T$ (1990) Target selection by cortical axons: alternative mechanisms to establish axonal connections in the developing brain. Cold Spring Harbor Symp Quant Biol 55:453-468.

Phelan KA, Hollyday M (1990) Axon guidance in muscleless wings: the role of muscle cells in motoneuronal pathway selection and muscle nerve formation. J Neurosci 10:2699-2716.
Rau MS, Escar y J, Sun Y, Perreau J, Patterson PH, Zigmond RE, Brulet P, et al. (1993) Leukemia inhibitory factor mediates an injury response but not a target-mediated developmental transmitter switch in sympathetic neurons. Neuron 11:1175-1185.

Rao MS, Jaszczak E, Landis SC (1994) Innervation of footpads of normal and mutant mice lacking sweat glands. J Comp Neurol 346: 613-625.

Schecterson LC, Bothwell M (1992) Novel roles for neurotrophins are suggested by BDNF and NT-3 mRNA expression in developing neurons. Neuron 9:449-463,

Schotzinger R, Landis SC (1988) Cholinergic phenotype developed by noradrenergic sympathetic neuorns after innervation of a novel cholinergic target in vivo. Nature 335:637-639.

Schotzinger R, Landis SC (1990) Acquisition of cholinergic and peptidergic properties by the sympathetic innervation of rat sweat glands requires interaction with normal target. Neuron 5:91-100.

Shelton D, Reichardt L (1984) Expression of the $\beta$ nerve growth factor gene correlates with the density of sympathetic innervation in effector organs. Proc Natl Acad Sci USA 81:7951-7955.

Tessier-Lavigne M, Placzek M, Lumsden AGS, Dodd J, Jessell T (1988) Chemotropic guidance of developing axons in the mammalian central nervous system. Nature 336:775-778.

Thoenen H, Barde Y-A (1980) Physiology of nerve growth factor. Physiol Rev 60:1284-1335.

Tosney KW (1987) Proximal tissues and patterned neurite outgrowth at the lumbosacral level of the chick embryo: deletion of the dermamyotome. Dev Biol 122:540-558.

Tyrrell S, Landis SC (1994) Disruption of target interactions prevents the development of enkephalin immunoreactivity in sympathetic neurons. J Neurosci 14:5708-5721.

Vos P, Stark F, Pittman R (1991) Merkel cells in vitro: production of nerve growth factor and selective interactions with sensory neurons. Dev Biol 144:281-300.

Voyvodic JT (1987) Development and regulation of dendrites in the rat superior cervical ganglion. J Neurosci 7:904-912.

Voyvodic IT (1989) Peripheral target regulation of dendritic geometry in the rat superior cervical ganglion. J Neurosci 9:1997-2010.

Yaginuma H, Oppenheim R (1991) An experimental analysis of in vivo guidance cues used by axons of spinal interneurons in the chick embryo: evidence for chemotropism and related guidance mechanisms. J Neurosci 11:2596-2613.

Yodlowski ML, Fredieu JR, Landis SC (1984) Neonatal 6-hydroxydopamine treatment eliminates cholinergic sympathetic innervation and induces sensory sprouting in rat sweat glands. J Neurosci $4: 1535-1548$. 